This document is the Accepted Manuscript version of a Published Work that appeared in final form in Macromolecules, copyright (c) 2018 American Chemical Society after peer review and technical editing by the publisher. To access the final edited and published work see https://doi.org/10.1021/acs.macromol.8b01313

\title{
Promotion of self-nucleation with latent Form I nuclei in polybutene-1 and its copolymer
}

Zefan Wang ${ }^{1,2}$, Xia Dong ${ }^{1,2}$, Dario Cavallo $^{3}$, Alejandro J. Müller ${ }^{* 4,5}$, Dujin Wang $* 1,2$

${ }^{1}$ CAS Key Laboratory of Engineering Plastics, CAS Research/Education Center for Excellence in Molecular Sciences, Institute of Chemistry, Chinese Academy of Sciences, Beijing 100190, China

${ }^{2}$ University of Chinese Academy of Sciences, Beijing 100049, China

${ }^{3}$ Department of Chemistry and Industrial Chemistry, University of Genova, via Dodecaneso, 31 - 16146 Genova, Italy

${ }^{4}$ POLYMAT and Polymer Science and Technology Department, Faculty of Chemistry, University of the Basque Country UPV/EHU, Paseo Manuel de Lardizabal 3, 20018 Donostia-San Sebastián, Spain

${ }^{5}$ Ikerbasque, Basque Foundation for Science, Bilbao, Spain

*Corresponding authors: djwang@iccas.ac.cn and alejandrojesus.muller@ehu.es 


\section{ABSTRACT}

The formation of Form I nuclei of polybutene-1 (PB-1) and its copolymer with polyethylene (PB1-ran-PE) has been studied by means of modified self-nucleation protocols. Even when the self-nucleation temperature was high enough and all Form II crystals melt, re-crystallization can be accelerated if the melt-crystallized sample was annealed at low temperatures (below $60^{\circ} \mathrm{C}$ for $\mathrm{PB}-1$ and $75^{\circ} \mathrm{C}$ for PB1-ran-PE) for just 3 min. These results suggest the formation of latent Form I nuclei within Form II crystals. This hypothesis is consistent with the observed growth of a small amount of Form I crystals during heating, after previous annealing at temperature lower than $20^{\circ} \mathrm{C}$. In addition, a peculiar phenomenon was found in PB1-ran-PE, as both Form II and Form I' can be induced by the presence of latent Form I nuclei, due to cross-nucleation and self-nucleation effects, respectively. The final ratio of the two kinds of crystal Forms is a result of the competition between the two nucleation rates, which strongly depend on crystallization temperature. In this work, we have shown that careful design of novel self-nucleation protocols can yield evidence of the early stages of Form II to Form I transition, even when the degree of transformed crystals is below the limit of detection of conventional techniques sensitive to crystalline order (DSC, WAXD, FTIR).

Keywords: Polybutene-1, Butene-1/ethylene copolymer, polymorphic transformation, self-nucleation, crystalline memory. 


\section{INTRODUCTION}

Different from small molecules, semi-crystalline polymers with long linear chains can display a relatively wide crystallization and melting temperature range. ${ }^{1-3}$ When polymer samples are partially melted, their crystal remains can serve as nucleating agent to accelerate the subsequent crystallization process. This phenomenon is called self-nucleation. Even the orientation of the crystals can be inherited from the initial one (i.e., cloning $)^{4,5}$. Additionally, after complete melting of polymeric crystals, some memory of the crystalline ordered structure can still survive, and the crystallization of the polymer can be enhanced by melt memory effects. ${ }^{6-11}$

Polybutene- 1 is a polyolefin with excellent mechanical properties and complex polymorphic behavior that has attracted much interest from both industry and academia. 12, 13 The tetragonal Form II with an 11/3 helix can be obtained by cooling the sample directly from the melt. The thermodynamically stable Form I, with a 3/1 helix, is usually generated from the kinetically favored Form II by aging the sample at room temperature for 10 days. ${ }^{14}$ In addition, another polymorph, Form I', which has a similar crystal structure to Form I can also be formed ${ }^{15}$. Generally, Form I' can be obtained by several methods, such as solution crystallization, ${ }^{16}$ blending with isotactic polypropylene ${ }^{15}$ or copolymerization with other monomers. ${ }^{17-19}$

The transformation mechanism of Form II to Form I has been investigated for several decades. On a molecular level, it has been usually considered that the chirality of the 11/3 helix of Form II does not change when it transforms to Form I, which is made of 3/1 helix. Besides that, both tetragonal Form II and trigonal Form I share a 
common (110) plane. ${ }^{14}$

Recently, the precise crystallographic refinement of Form I and Form II have been made by Tashiro et al. ${ }^{20} \mathrm{~A}$ kinetic model was proposed suggesting that a transient hexagonal structure made of 11/3 helices developed first from Form II and then transform to stable Form I by 11/3 to 3/1 helices chain conformational changes. This mechanism can explain the formation of the twin structure of Form I observed by electron diffraction. Another interesting phenomenon is that the solid-solid transformation can be accelerated greatly if PB-1 samples are precooled to $-20{ }^{\circ} \mathrm{C}$ for several minutes. ${ }^{12}$

Men et al. designed an experiment to quantify the Form II to Form I transformation rate. $^{21,22}$ They demonstrated that a classic nucleation and growth model could be fitted to the Form II to Form I transition using the Tammann's two stage crystal nuclei development method, in analogy with small molecules. In addition, it was proposed that the transition mechanism could be promoted by the internal stress in the sample which is caused by an unbalanced shrinkage of amorphous and crystalline phases. However, the nucleation of Form I at the early stage of the transition has just been indirectly inferred from the acceleration of the overall transformation rate, and not directly evidenced.

Due to the complex polymorphism of PB-1, several researchers have tried to explore the self-nucleation behavior of PB-1 with different crystal modifications. Cavallo et al. ${ }^{23,24}$ found that Form II can be nucleated on the surface of Form I spherulites. This is an example of cross-nucleation. Furthermore, employing 
differential scanning calorimetry (DSC) and in situ Fourier transformation infrared microspectroscopic imaging, a small amount of Form I' was found directly inside Form I spherulites, when Form I crystals are partially molten. ${ }^{25-27}$ Men et al. investigated the melt memory effect of a butene-1/ethylene sample with high content of ethylene comonomer and found that Form I' can be formed directly from the heterogeneous melt, in a specific temperature range, as a consequence of melt heterogeneities produced by crystallizable chain sequence segregation coming from previous crystallization. It is proposed that the size of the segregated domains containing long crystallizable sequences is a function of the melt-annealing temperature, and thus the formation of either Form I' or Form II' depends on the interplay between this domain size and the critical nucleus size of the respective polymorph..$^{28-30}$

In this work, modified self-nucleation (SN) protocols were used as a novel method to detect the early stages of Form II-to-Form I transition in PB-1 and its copolymer. By annealing the melt-crystalized Form II crystals at low temperature for $3 \mathrm{~min}$, an enhanced nucleation was observed during the re-crystallization process, even for SN temperature high enough to completely melt all Form II crystals. These results indicated that a small amount of latent Form I nuclei, undetectable by FTIR and X-ray diffraction, formed during the annealing at low temperature. Different from PB-1, both Form I' and Form II can be induced by transformed Form I crystals in PB1-ran-PE, due to the competition between self-nucleation and cross-nucleation effects. Our work provides a sensitive method to investigate the early stage of the solid-solid transition in polymer crystals and sheds a new light on the understanding the structural change in Form II to 
Form I transition of PB-1 and its copolymer.

\section{EXPERIMENTAL SECTION}

\section{Materials.}

An isotactic polybutene-1 homopolymer (PB-1, trade name: PB0110M) and a butene-1/ethylene statistic copolymer (PB1-ran-PE, trade name: PB8340M) were purchased from Lyondell Basell Industries. The physical properties of them are listed in Table 1.

Table 1. Characterization of $P B-1$ and PB1-ran-PE samples.

\begin{tabular}{|c|c|c|c|c|c|c|c|}
\hline & & Melt flow & $\mathrm{M}_{\mathrm{w}}$ & PDI & Content of & $\mathrm{T}_{\mathrm{m}, 1}\left({ }^{\circ} \mathrm{C}\right.$, & $\mathrm{T}_{\mathrm{m}, 2}\left({ }^{\circ} \mathrm{C}\right.$, \\
\hline & Trade & rate (MFR) & $(\mathrm{kg} / \mathrm{mo}$ & & comonomer & Form I) & Form II) \\
\hline & name & $\left(190^{\circ} \mathrm{C} / 2.16\right.$ & l) & & (Characteriz & & \\
\hline & & $\mathrm{kg})$ & & & ed by & & \\
\hline & & & & & NMR $)^{31}$ & & \\
\hline PB-1 & PB0110M & 0.4 & 65.7 & 3.52 & 0 & 128 & 117 \\
\hline PB1- & PB8340M & 4 & 28.1 & 4.25 & $4.8 \%$ & 113 & 97 \\
\hline$-\mathrm{P}$ & & & & & & & \\
\hline
\end{tabular}

\section{Differential Scanning Calorimeter (DSC).}

The thermal and the self-nucleation behavior of the PB-1 and PB1-ran-PE were characterized by a TA differential scanning calorimeter (Q2000) under nitrogen atmosphere (50 ml/min). The instrument was calibrated with indium. 
The thermal protocols applied in this work can be divided in two stages: sample preparation and self-nucleation.

(a) Sample preparation stage. As indicated in Figure 1, the samples were first heated to $200{ }^{\circ} \mathrm{C}$ for 3 min to erase thermal history, followed by quenching to $T_{c, 1}$ for $90 \min \left(T_{c, 1}\right.$ was set to $85{ }^{\circ} \mathrm{C}$ for PB-1 and $75{ }^{\circ} \mathrm{C}$ for PB1-ran-PE). In this way, pure Form II was obtained (see Figure 1a and 1b). In order to investigate the structural changes during the annealing process at low temperatures, some of the samples were cooled to different lower treatment temperatures $\left(T_{l}\right)$ at $10{ }^{\circ} \mathrm{C} / \mathrm{min}$, then they were kept at these low temperatures for 3 min (Figure 1c and 1d).

(b) Self-nucleation stage. Two types of SN procedures were designed and implemented:

(b-1) Figure 1a and 1c show standard SN protocols similar to those commonly used in the literature (see for instance ref. 2). Samples were heated to different SN temperatures (or $T_{s}$ temperatures) for $5 \mathrm{~min}$, and then cooled to $30^{\circ} \mathrm{C}$ for 3 min, followed by heating to $200{ }^{\circ} \mathrm{C}$. Both cooling and heating rates were 10 ${ }^{\circ} \mathrm{C} / \mathrm{min}$.

(b-2) In order to investigate the possible competition between self-nucleation and cross-nucleation of PB1-ran-PE, another SN experiment was designed and termed “isothermal $\boldsymbol{S N}$ ” (see Figure $1 \mathrm{~b}$ and d). After the preparation stage (i.e., b-1 above), the samples were heated to a $T_{s}$ temperature of $118{ }^{\circ} \mathrm{C}$ for $5 \mathrm{~min}$. At this temperature, only Form II can melt but any Form I that could have 
formed (by transformation of Form II) will remain. After that, all samples were quenched to different $T_{c, 2}$ temperatures for $120 \mathrm{~min}$ and finally heated to 200 ${ }^{\circ} \mathrm{C}$, also at $10{ }^{\circ} \mathrm{C} / \mathrm{min}$.

According to Lotz et al. ${ }^{32}$, three different SN temperature Domains can be defined: (1) Domain I or complete melting domain occurs when the applied $T_{s}$ temperatures are high enough to melt all crystals and erase crystalline memory, so that isotropic melts are obtained. In this $T_{s}$ range, the peak crystallization temperature of the material during cooling from $T_{s}$ remains constant. (2) In Domain II, $T_{s}$ is high enough to melt all crystals in the sample or most of them, leaving self-nuclei constituted by ordered regions that remember their crystalline state or by small unmolten crystal fragments respectively (see ref. 2). The crystallization temperature of polymer greatly increases as $T_{s}$ decreases in Domain II. (3) In Domain III, $T_{s}$ values are too low and produce partial melting. Unmolten polymer crystals anneal during the $5 \mathrm{~min}$ at $T_{\mathrm{s}}$ and a second melting peak is normally observed in the subsequent heating curves after SN. 

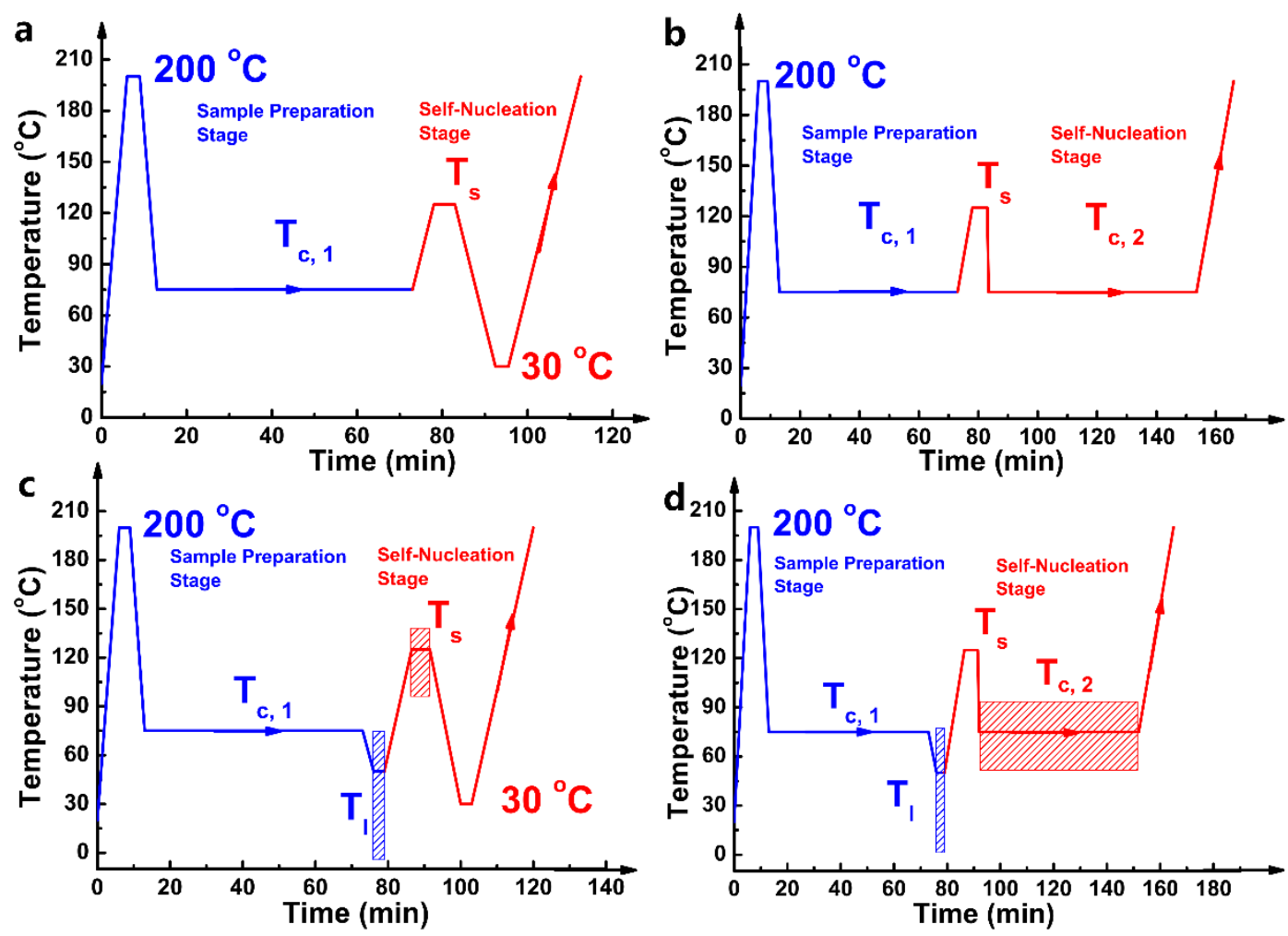

Figure 1. The non-isothermal $(a, c)$ and isothermal $(b, d) S N$ experimental protocols designed and implemented in this work (see text). Samples with different polymorphic contents are prepared without annealing $(a, b)$ or with an annealing step at low temperatures $(c, d)$.

\section{Polarized Light Optical Microscopy (PLOM).}

The morphology of the self-nucleation and cross-nucleation behavior were observed in situ by an Olympus BX51 polarized optical microscopy equipped with a Canon 40D camera system. The thermal treatment was carried out by a calibrated Linkam 600 hot stage. Before the observation, the pellets of PB-1 and PB1-ran-PE samples were hot pressed into films with thickness of approximately $80 \mu \mathrm{m}$ and sandwiched in between two glass slides. Thermal treatments were consistent with the DSC SN protocols. 


\section{Wide Angle X-ray Diffraction (WAXD).}

The crystal structure of the samples was characterized by wide angle X-ray diffraction (WAXD), using a Xeuss 2.0 system of Xenocs (France) with a Cu Ka source (wavelength $=1.54 \AA$ ). The sample to detector distance is $140.2 \mathrm{~mm}$. Scattering patterns were collected by a semiconductor detector (Pilatus, 300K DECTRIS, Swiss) with a

resolution of $487 \times 619$ pixels (pixel size $=172 \mu \mathrm{m}^{2}$ ). Temperature control was applied with a Linkam TST 350 hot stage. Each WAXD pattern was collected for 5 min and the one-dimensional intensity profiles were integrated using the standard procedure with background subtraction. The main reflections at $11.9^{\circ}, 16.9^{\circ}$ and $18.5^{\circ}$ are assigned to (200), (220) and (213+311) planes of Form II. Peaks at $10.0^{\circ}, 17.5^{\circ}$ and $20.4^{\circ}$ belong to (110), (130) and (220+211) crystal planes of Form I and I' respectively. ${ }^{24,28}$

\section{Fourier Transform Infrared Spectroscopy (FTIR).}

In situ FTIR spectra were obtained by using a Nicolet 6700 FTIR spectrometer equipped with a Linkam FIIR600 hot stage. The films of PB-1 and PB1-ran-PE were first heated to $200{ }^{\circ} \mathrm{C}$ for $5 \mathrm{~min}$ followed by a quenching to $T_{c, 1}$ for $90 \mathrm{~min}$. After that, samples were then cooled from $T_{c, 1}$ to $0{ }^{\circ} \mathrm{C}$ for $3 \mathrm{~min}$ and heated to $80{ }^{\circ} \mathrm{C}$ again. Both cooling and heating rates were $3{ }^{\circ} \mathrm{C} / \mathrm{min}$. During this process, FTIR spectra were obtained with a resolution of $4.0 \mathrm{~cm}^{-1}$ and 16 scans with $1{ }^{\circ} \mathrm{C}$ intervals. The baseline of each spectrum was corrected according to the standard procedure. ${ }^{33,} 34$ Two typical strong absorption bands at $923 \mathrm{~cm}^{-1}$ and $905 \mathrm{~cm}^{-1}$ are usually adopted to differentiate 
Form I and Form II crystals respectively. ${ }^{27,}$ 35, 36

\section{Results and Discussion}

The self-nucleation behavior of PB-1 homopolymer with or without annealing at low temperatures.

Figure 2 shows the non-isothermal SN (i.e., the SN protocol described in Figure 1a, see experimental) results for $\mathrm{PB}-1$ at a $T_{s}=128{ }^{\circ} \mathrm{C}$, with or without annealing at $0{ }^{\circ} \mathrm{C}$ for $3 \mathrm{~min}$.
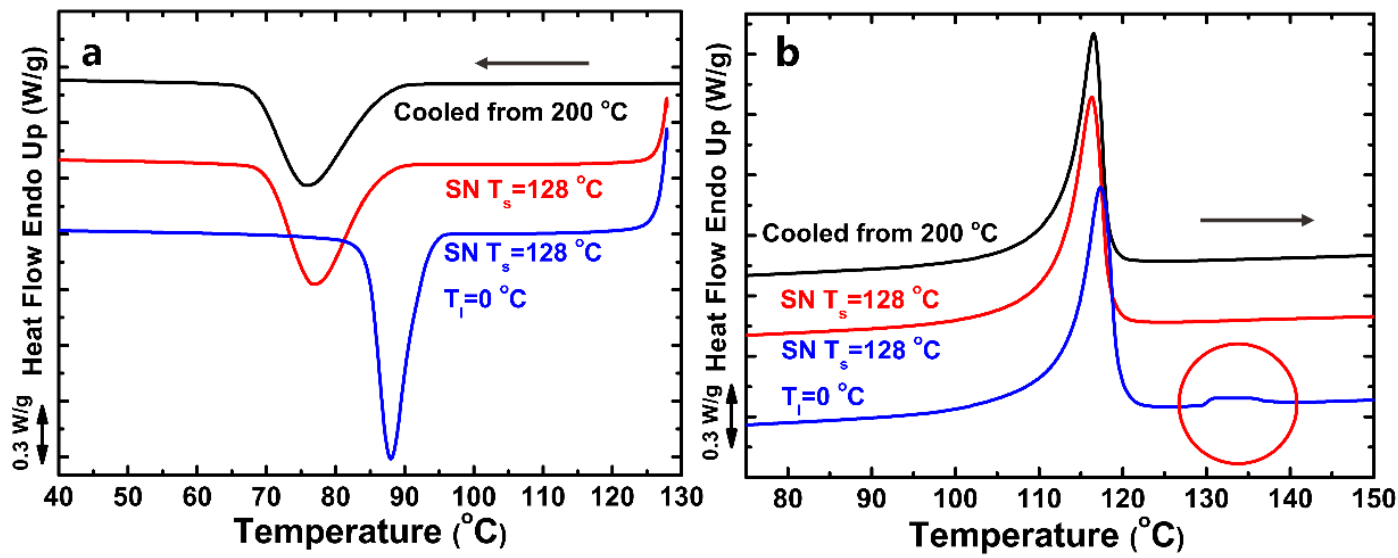

Figure 2. DSC cooling (a) and subsequent heating (b) curves for the PB-1 samples selfnucleated at $T_{s}$ temperature at $10^{\circ} \mathrm{C} / \mathrm{min}$. Samples with different polymorphisms were compared: pure Form II crystallized at $85{ }^{\circ} \mathrm{C}$ isothermally (red); Form II with small amount of Form I crystals annealed at $0{ }^{\circ} \mathrm{C}$ for 3 min after isothermal crystallization at $85^{\circ} \mathrm{C}$ (blue).

In a standard $\mathrm{SN}$ protocol, the sample is cooled from $T_{S}$ down to a temperature low enough to allow the full crystallization of the material. The situation in PB-1 is 
complicated by its polymorphic transformations. If the sample is cooled from $T_{\mathrm{s}}=128$ ${ }^{\circ} \mathrm{C}$ (a temperature high enough to completely melt Form II) to $30^{\circ} \mathrm{C}$, PB-1 Form II can completely recrystallize. Hence, if the sample is kept at $30{ }^{\circ} \mathrm{C}$ for a short time (3 min) and then reheated, the DSC trace drawn in red in Figure 2 is obtained.

The peak crystallization temperature of the sample self-nucleated at $T_{s}=128{ }^{\circ} \mathrm{C}$ (without any annealing at $0{ }^{\circ} \mathrm{C}$ ) is located at $76^{\circ} \mathrm{C}$, which is nearly same as that of the sample directly cooled from $200{ }^{\circ} \mathrm{C}$. In the other words, all the crystals and crystalline memory of Form II are destroyed at this $T_{s}$ value. ${ }^{2}$

Performing a small modification to the thermal protocol described as standard SN for PB-1 Form II, the sample is cooled to $0^{\circ} \mathrm{C}$ and kept at that temperature for $3 \mathrm{~min}$, a very short time, considering that Form II can only completely transformed to Form I after days at room temperature. However, the peak crystallization temperature of the sample, annealed at $0{ }^{\circ} \mathrm{C}$ for $3 \mathrm{~min}$ after $\mathrm{SN}$ at $T_{s}=128{ }^{\circ} \mathrm{C}$, increased to $88^{\circ} \mathrm{C}$ (from $76^{\circ} \mathrm{C}$ ), and the crystallization exotherm becomes narrower. This increase in $T_{c}$ must correspond to a nucleating effect on Form II crystals.

Figure $2 \mathrm{~b}$ shows that besides the expected melting peak of Form II at $117^{\circ} \mathrm{C}$, a very small endothermic peak within the $130{ }^{\circ} \mathrm{C}$ to $136{ }^{\circ} \mathrm{C}$ range, that corresponds to Form I, can also be appreciated in the heating scan of the sample annealed at $0{ }^{\circ} \mathrm{C}$. These transformed crystals are then responsible for the nucleation effect caused on the crystallization of PB-1 as Form II crystals during cooling from $T_{s}$. Figure $\mathrm{S} 1$ shows that both FTIR and WAXD could not detect any Form I crystals during the cooling process. However, when the sample was heated to $80{ }^{\circ} \mathrm{C}$ again, a slight enhancement of the 923 
$\mathrm{cm}^{-1}$ band was clearly observed, especially in the second derivative spectra (Figure 3). These results indicate that the small amount of Form I crystals observed by DSC are actually formed during the heating process, where the growth of Form I crystals within form II phase is faster. ${ }^{21}$ According to Tammann's two-stage nucleation theory and the classical nucleation and growth theory proposed by Men et al. ${ }^{21}$, annealing at low temperatures can cause an unbalanced shrinkage of amorphous and crystalline phases which may accelerate the formation of Form I crystals.
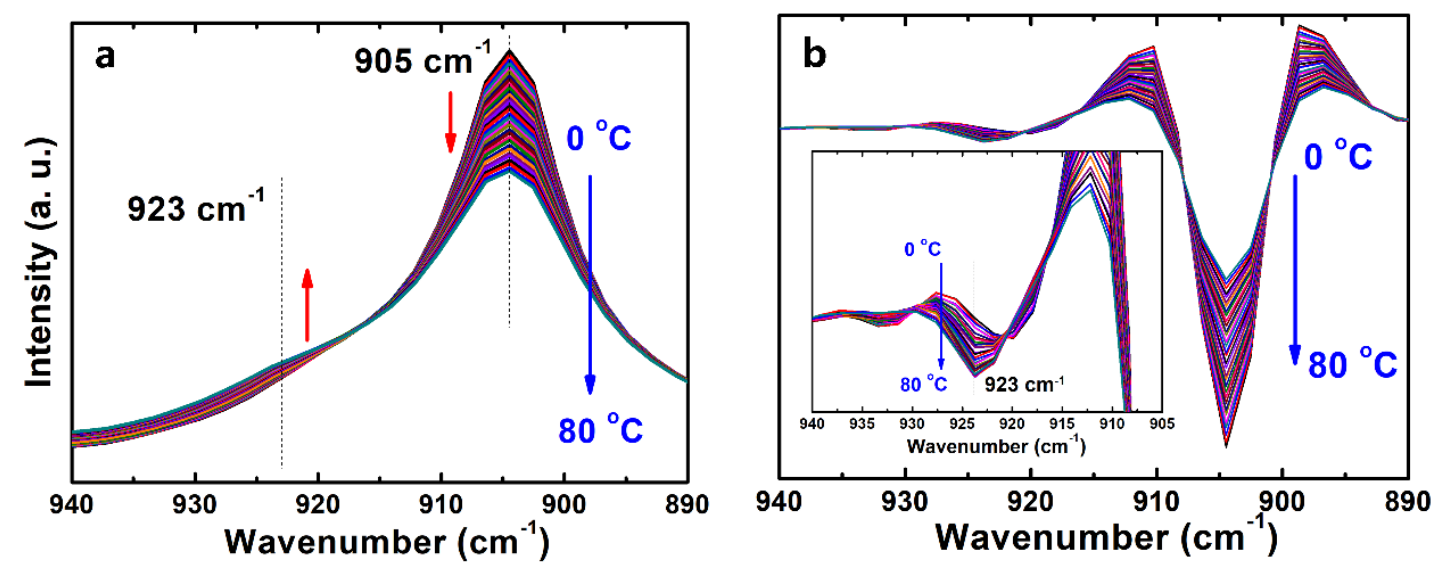

Figure 3. Temperature-resolved FTIR spectra (a) and the corresponding second derivative spectra (b) of PB-1 sample during the heating process. The sample was isothermally crystallized from the melt at $85{ }^{\circ} \mathrm{C}$, then it was cooled to $0{ }^{\circ} \mathrm{C}$ for $3 \mathrm{~min}$, and finally heated to $80^{\circ} \mathrm{C}$ at $3{ }^{\circ} \mathrm{C} / \mathrm{min}$ while FTIR spectra were recorded.

Similar results were also obtained by PLOM (Figure S2). The morphology of PB1 spherulites did not change after annealing at $0{ }^{\circ} \mathrm{C}$, as compared to that obtained when crystallizing at $85{ }^{\circ} \mathrm{C}$ (Figure S2 a and b). However, when the PB-1 film was heated to $128{ }^{\circ} \mathrm{C}$, all Form II crystals melted but a weakly birefringent template remained at 
temperatures above the melting point of Form II crystals. By comparing the PLOM results to the results of DSC measurements (Figure 2), it can be deduced that this template must belong to the small fraction of Form I crystals obtained during the heating process. After that, the sample was quenched to $85{ }^{\circ} \mathrm{C}$ and $\mathrm{PB}-1$ was able to crystallize very fast, within a few minutes, because of the high nucleation density produced by the Form I crystals that survived. In this situation, only Form II is nucleated by the Form I template, i.e., a cross-nucleation effect. ${ }^{23}$

The effect of annealing at low temperatures: the formation of Form I latent nuclei.

The Form II to Form I transformation mechanism of PB-1 crystals has been investigated for several decades since the introduction of PB-1. ${ }^{12,14,20}$ According to the literature, the transformation rate at room temperature is faster than at lower or higher temperatures. Precooling at temperatures below $0{ }^{\circ} \mathrm{C}$ has also been used to accelerate the transformation process. Both FTIR and WAXD were used to attempt the detection of structural changes of Form II during the cooling process (Figure S1a, S1c and S1e). However, no signals corresponding to Form I could be detected, indicating that these techniques are not sensitive to the presence of Form I nuclei, due to their low volume fraction under the applied conditions.

Self-nucleation can be an effective way to detect the presence of active nuclei, even if they are present in small quantities. ${ }^{2}$ The results of the non-isothermal SN tests with annealing at different low temperatures (using the protocol outlined in Figure 1c, see experimental) are shown in Figure 4. 
According to the applied sample preparation stage protocol (see experimental), the PB-1 sample is first molten at $200{ }^{\circ} \mathrm{C}$, then isothermally crystallized at $85^{\circ} \mathrm{C}$ into Form II. Next, the sample is cooled down to a temperature denoted $T_{l}$ (see Figure $1 \mathrm{a}$ ), where it is annealed at low temperatures for just 3 minutes, before it is self-nucleated employing a constant $T_{s}=128{ }^{\circ} \mathrm{C}$. If $T_{l}$ is higher or equal to $60{ }^{\circ} \mathrm{C}$, the behavior of the sample is the same to that of a sample cooled from $200{ }^{\circ} \mathrm{C}$ directly. Therefore, such relatively high $T_{l}$ temperatures (60 ${ }^{\circ} \mathrm{C}$ and higher) do not influence the sample crystal structure, which remains as Form II exclusively. 

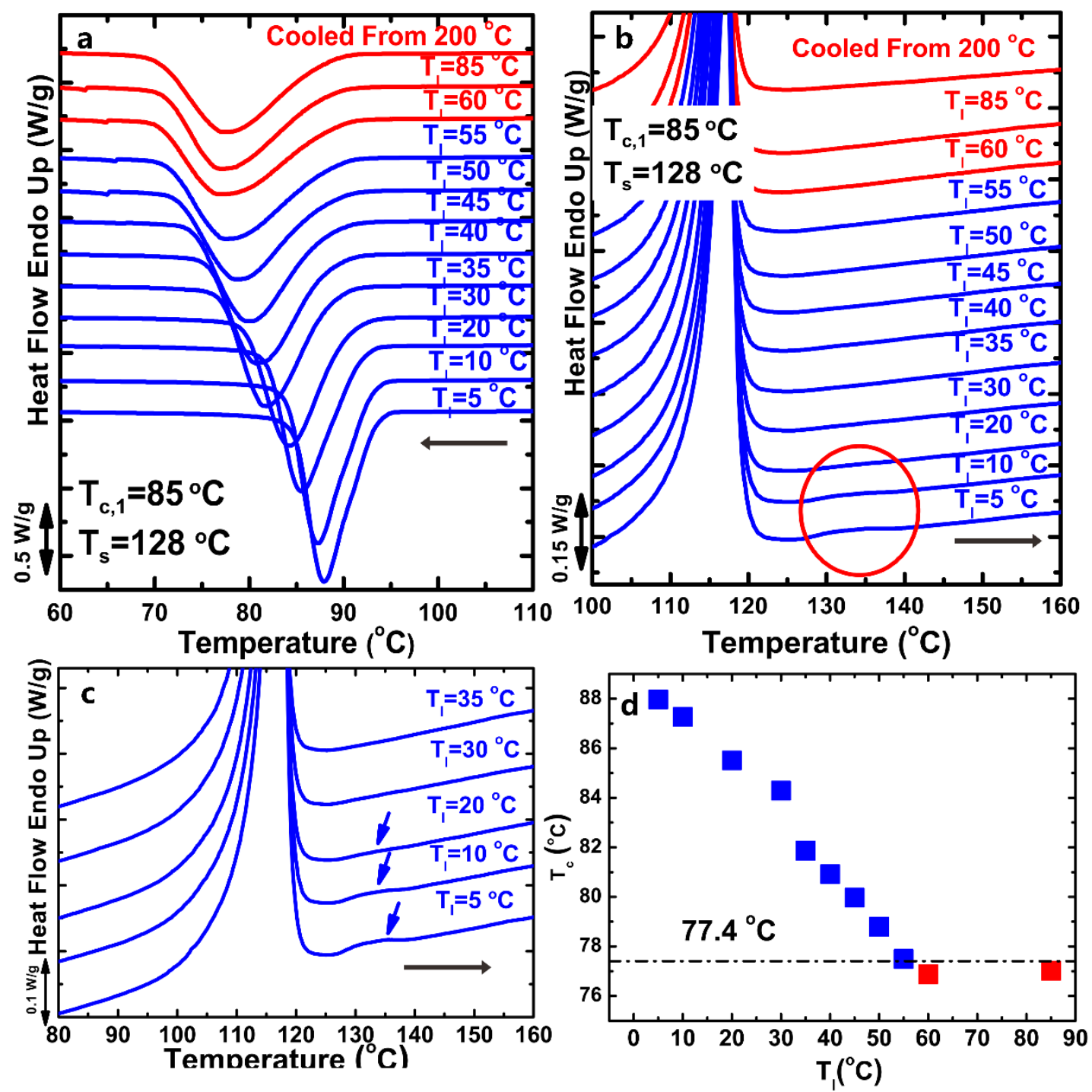

e

Form I latent nuclei

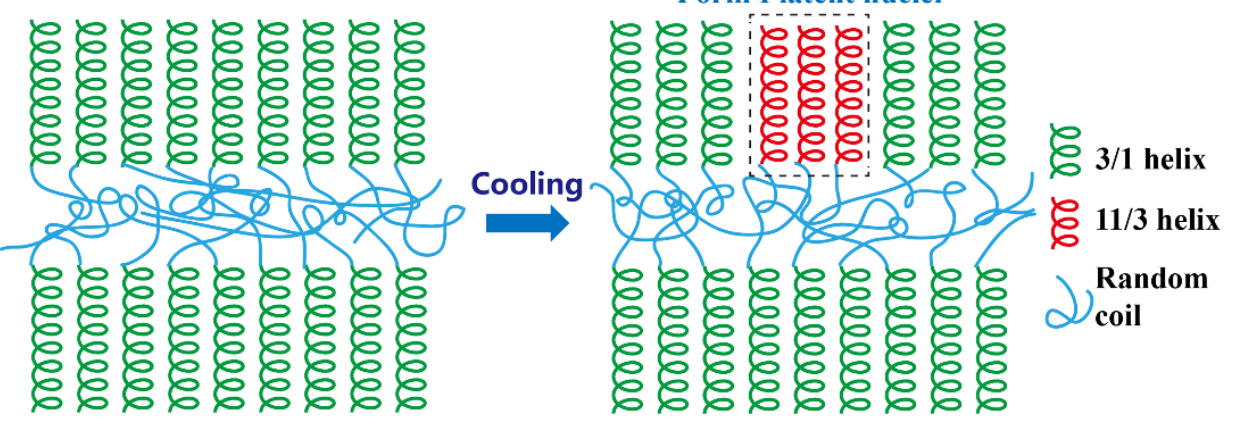

Figure 4. The effect of annealing at low temperatures on the self-nucleation of PB-1. (a) DSC cooling scans from a SN temperature of $128^{\circ} \mathrm{C} ;(b, c)$ subsequent heating scans. Red and blue curves demarcate the Region I and Region II, respectively. The DSC curve corresponding to the crystallization from an isotropic melt (i.e., cooled from $200^{\circ} \mathrm{C}$, a temperature at which thermal 
history is erased is presented for comparison purposes); (d) annealing temperature vs. crystallization temperature; the horizontal line refers to the crystallization temperature of PB1 after erasing thermal history. (e) scheme for formation of the Form I nuclei.

When annealing temperatures (i.e., $T_{l}$ temperature values) lower than $60{ }^{\circ} \mathrm{C}$ are employed, the peak crystallization temperature $\left(T_{c}\right)$, after $\mathrm{SN}$ at $T_{S}=128{ }^{\circ} \mathrm{C}$, increases with respect to the standard behavior of PB-1 upon cooling from $200{ }^{\circ} \mathrm{C}$, as shown in Figure $4 \mathrm{a}$. As the $\mathrm{T}_{\mathrm{c}}$ value is proportional to the nucleation density, this result indicates that the low temperature annealing is producing a nucleation effect. The subsequent heating scans, recorded immediately after the cooling traces shown in Figure 4a, are presented in Figure 4b. When the $T_{l}$ temperature approaches $20^{\circ} \mathrm{C}$, a small melting peak located between $130{ }^{\circ} \mathrm{C}$ and $140{ }^{\circ} \mathrm{C}$ can be observed. This additional melting peak at higher temperatures is due to the melting of a small fraction of Form I crystals.

The behavior of the sample after low temperature annealing followed by SN presented in Figure 4a and $4 \mathrm{~b}$ can be interpreted as follows. When $T_{l}$ values lower than $60{ }^{\circ} \mathrm{C}$ but higher than $20^{\circ} \mathrm{C}$ are employed, some Form I nuclei are produced during the 3 min at $T_{l}$ within the existing Form II crystals. However, their presence can only be detected by the subsequent nucleation effects (the increase in $T_{c}$ values reported in Figure 4a). In this case, these nuclei are termed "latent nuclei”. ${ }^{37-39}$ Nevertheless, when $T_{l}$ is $20{ }^{\circ} \mathrm{C}$ or lower, Form I nuclei are able to grow into crystals during the heating process, and they can be detected by their melting endotherm (at $130-140{ }^{\circ} \mathrm{C}$ ) in Figure 4b. The $T_{c}-T_{l}$ relationship is summarized in Figure $4 \mathrm{~d}$. According to the $T_{c}$ value of the 
self-nucleated sample, two temperature Regions can be defined: Region I (above $55^{\circ} \mathrm{C}$ ), where only Form II exists in the sample without any Form I nuclei and Region II (below $55^{\circ} \mathrm{C}$ ), where latent Form I nuclei are generated, thereby nucleating the formation of Form II crystals.

These results confirm earlier work by Men et al., ${ }^{21}$ indicating that the nucleation of Form II by Form I nuclei (and/or crystals) strongly depends on annealing the sample at low temperatures. A simplified scheme is shown in Figure 4e. The loose 11/3 helices in Form II may be transformed to compact 3/1 helices during annealing at low temperatures for a short time, then the packing mode of the polymer chain can change and provide nuclei for the following melt-crystallization of Form II, as shown for fullytransformed Form I samples self-nucleated at relatively high temperatures. ${ }^{23,24}$

According to the above discussion, even a small amount of Form I crystals (or nuclei) generated at low temperatures can have a large effect on the self-nucleation of PB-1. Therefore, it is necessary to investigate the effect of different $T_{s}$ values for PB-1 Form II with or without Form I nuclei (Figure 5).

The three standard SN Domains can be observed for pure Form II if the sample is crystallized at $85{ }^{\circ} \mathrm{C}$ without low temperature annealing (following the $\mathrm{SN}$ protocol described in Figure 1a). When the $T_{s}$ value reaches $122{ }^{\circ} \mathrm{C}$, all the ordered structures are destroyed and the melt reaches an isotropic state (Domain I). The range of Domain II $\left(117 \sim 122{ }^{\circ} \mathrm{C}\right)$ is relatively narrow (see Figure 5e), like in other non-polar homopolymers, such as polyethylene ${ }^{7}$ or polypropylene. ${ }^{32}$

In the case that PB-1 samples are annealed at low temperatures to induce the 
formation of Form I crystals (annealing at $0{ }^{\circ} \mathrm{C}$ for $3 \mathrm{~min}$, using the $\mathrm{SN}$ protocol presented in Figure 1c), the situation is more complex. Above $136{ }^{\circ} \mathrm{C}$, all crystals are molten (both Form II and Form I) and the melt becomes isotropic (Domain I in Figure $5 f)$.

As the PB-1 samples annealed at low temperature contain both Form I and Form II crystals, the SN Domains can be defined for each polymorph (as their melting points are different). When the $T_{s}$ value is below $136{ }^{\circ} \mathrm{C}$, PB-1 Form II is nucleated by unmolten Form I crystals by cross-nucleation, see how the $T_{c}$ values increase in Figure 4b with respect to samples in Domain I. Therefore, in the range between $T_{s}$ temperatures of 136 and $128^{\circ} \mathrm{C}$, a Domain II (Form II) can be defined for the cross-nucleation of Form II by Form I crystals. However, in the same temperature range, the 5 min holding time at $T_{s}$ during SN, also produces annealing of the Form I crystals present in the sample. The annealing that occurs in that temperature range is highlighted with a circle and with a close up with arrows in Figure 5d. Therefore, in the same temperature range of 136

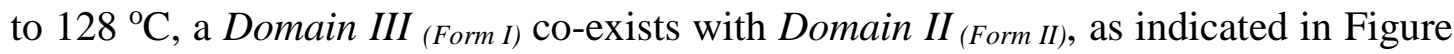
$5 f$. 

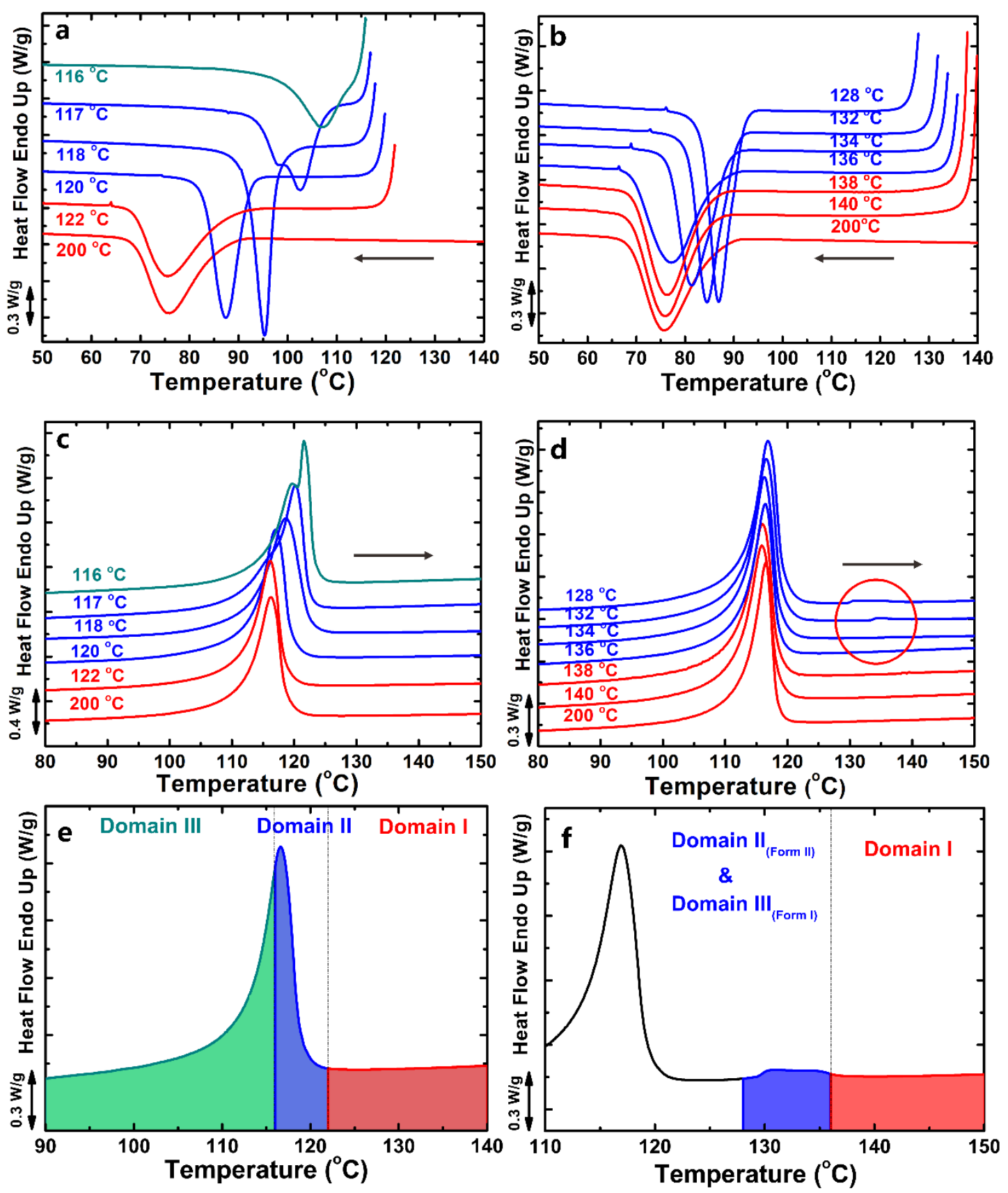

Figure 5. Self-nucleation behavior of PB-1 containing only Form II $(a, c, e)$ or Form II mixed with a small amount of Form I crystals (or nuclei) $(b, d, f) .(a, b)$ DSC cooling scans from different $T_{s}$ temperatures; subsequent heating process $(c, d) ;(e, f)$ representations of the $S N$ domains for PB-1 samples with different polymorphic structures. 
Self-nucleation of butene-1/ethylene statistic copolymer: the formation of Form I' crystals.

Figure 6 shows the self-nucleation behavior results (after application of the SN protocols shown in Figure 1b and d) of PB1-ran-PE samples with different thermal histories. For the sample crystallized at $75{ }^{\circ} \mathrm{C}$ without annealing at $0{ }^{\circ} \mathrm{C}$, all the crystals have completely disappeared at $118^{\circ} \mathrm{C}$ (see the optical micrograph in Figure 6b), which means that the sample contains only Form II crystals. In this situation the DSC heating trace shows a single melting peak at $102{ }^{\circ} \mathrm{C}$, corresponding to the melting point of Form II crystals (Figure 6a).

Different results are obtained if the PB1-ran-PE copolymer is annealed at $0{ }^{\circ} \mathrm{C}$ for 3 min before self-nucleation. A small melting peak corresponding to Form I appears in the DSC heating scan at $120^{\circ} \mathrm{C}$ (Figure 6a, labeled with an arrow). Additionally, when the sample with an identical thermal protocol is observed by PLOM at $118{ }^{\circ} \mathrm{C}$, a template of Form I crystals can be observed (Figure 6b and S3). Similar to the homopolymer case, the formation of Form I crystals can be attributed to their nucleation at $0{ }^{\circ} \mathrm{C}$, and their growth during the subsequent heating process, facts that were verified by FTIR results (Figure S1 and Figure S3). The above observations by DSC and PLOM regarding the production of a small fraction of Form I crystals parallel the results obtained in PB-1 homopolymer.

However, a difference with respect to PB-1 homopolymer can be observed in Figure 6a, as a large amount of PB-1 Form I' crystals and a small quantity of Form II crystals are produced upon re-crystallization at $75{ }^{\circ} \mathrm{C}$ (applying the protocol described 
in Figure 1d).

The morphology was also observed by PLOM (Figure 6b bottom right). During the isothermal crystallization process at $75^{\circ} \mathrm{C}$, the birefringence of the Form I template was largely enhanced because of the generation of Form I' caused by homo-polymorphic self-nucleation. Additionally, some bright spots (signaled with arrows) that belong to Form II were generated by the cross-nucleation effect. These results indicate that Form I' can be induced when there are residual Form I crystals in PB1-ran-PE.
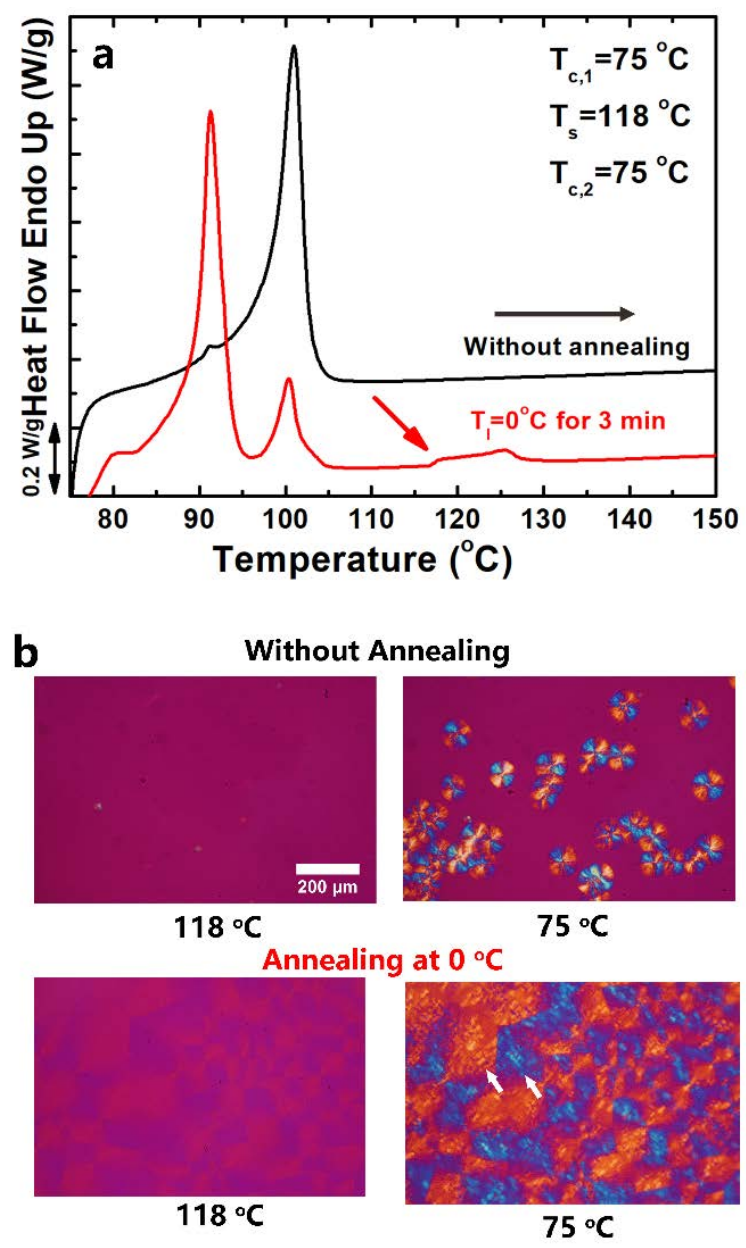

Figure 6. The self-nucleation behavior of PB1-ran-PE sample with or without annealing at 0 
${ }^{\circ} \mathrm{C}$. The $T_{s}$ value is $118{ }^{\circ} \mathrm{C}$. All the samples were first crystallized at $75{ }^{\circ} \mathrm{C}$ after erasing their thermal history. (a) DSC heating scans for the self-nucleated sample at $10{ }^{\circ} \mathrm{C} / \mathrm{min}$; (b) corresponding PLOM micrographs for the samples self-nucleated at $118{ }^{\circ} \mathrm{C}$ (left) and crystallized at $75^{\circ} \mathrm{C}$ isothermally (right).

Comparing to PB-1 homopolymer Form I self-nucleation results by Cavallo et al. ${ }^{26}$, it seems that the inclusion of ethylene comonomer enhances the nucleation of Form I' crystals. According to previous studies, ${ }^{15}$ two requirements are needed for the formation of Form I' crystals in PB-1 homopolymer: a hindrance to the nucleation of the fastergrowing Form II polymorph and the presence of an interface to selectively nucleate Form I' crystals. In PB1-ran-PE copolymer, a small amount of Form I crystals can efficiently induce the formation of Form I' crystals, at the expense of Form II crystals which compete for the crystallization of the amorphous fraction. Therefore, it must be deduced that either the growth rate of the two polymorphs is comparable in the copolymer sample, or that the efficiency and selectivity of Form I crystals towards Form I' nucleation overwhelm Form II heterogeneous nucleation. 

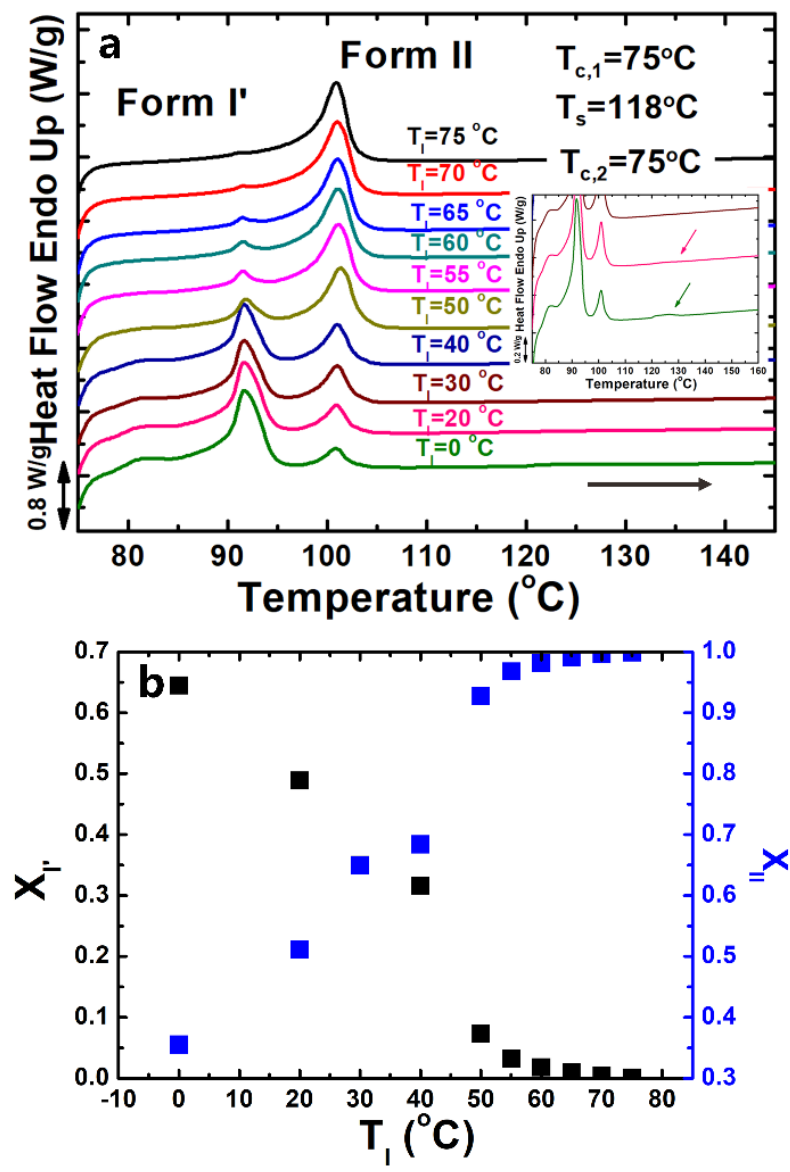

Figure 7. The effect of low annealing temperatures on the self-nucleation of PB1-ran-PE. The SN temperature is $118{ }^{\circ} \mathrm{C}$. All the samples were first crystallized at $75{ }^{\circ} \mathrm{C}$ after erasing their thermal history (according to the SN protocol presented in Figure 1d). (a) DSC heating scan of the self-nucleated sample after crystallizing at $75^{\circ} \mathrm{C}$; (b) summary of the content of Form $I^{\prime}$ and Form II crystals according to DSC results.

In analogy with previous results for the homopolymer sample, the production of Form I latent nuclei by low temperature annealing could not be evidenced by FTIR or WAXD in the copolymer (Figure S1b, S1d and S1f). Therefore, we turned once more to self-nucleation in order to detect the production of Form I' nuclei and crystals. 
Instead of the non-isothermal SN procedure used in the homopolymer, a different SN protocol was applied for PB1-ran-PE, as described in the experimental section, Figure 1d, due to the strong melt memory effect in the copolymer. The sample was first isothermally crystallized at $75^{\circ} \mathrm{C}$, after erasing its thermal history by heating to $200{ }^{\circ} \mathrm{C}$. Then the sample was cooled to $T_{l}$ for 3 minutes, to perform the low temperature annealing process. After that, the sample was subjected to a modified SN process: first it was self-nucleated at $T_{s}=118{ }^{\circ} \mathrm{C}$ for 5 minutes and then cooled to $T_{c, 2}=75{ }^{\circ} \mathrm{C}$ for 70 min to isothermally crystallize. The formation of Form I' crystals during this isothermal crystallization process can be used to detect the nucleation of Form I within Form II crystals during the low temperature annealing process.

As shown in Figure 7a, even the sample crystalized at $75{ }^{\circ} \mathrm{C}$ without annealing displays a minor melting peak at $93{ }^{\circ} \mathrm{C}$ in the final heating trace. These results show that some amount of Form I' can be induced after SN process. Under the assumptions outlined above, i.e., that Form I' is nucleated in the presence of Form I crystals as a substrate, these results can be interpreted as a consequence of the presence of a small amount of Form I crystals, transformed directly from Form II during the first isothermal crystallization process at $75{ }^{\circ} \mathrm{C}$ (i.e., the preparation stage indicated in the protocol outlined in Figure 1d).

When the annealing temperature decreases from $70{ }^{\circ} \mathrm{C}$ to $0{ }^{\circ} \mathrm{C}$, a higher content of Form I' can be induced in the copolymer, while a concomitant reduction of Form II can be observed (Figure 7b). This behavior is indicative of an increased formation of Form I nuclei at lower annealing temperatures, which provides Form I'-selective nucleation 
sites during the subsequent re-crystallization. When the annealing temperature is below $20{ }^{\circ} \mathrm{C}$, a small endothermic peak at $125{ }^{\circ} \mathrm{C}$ can be observed in the final heating scan, corresponding to the melting of Form I crystals.

Besides the documented Form I' crystallization induced by suitable substrates, like Form I for PB-1 or iPP $\alpha$-form, ${ }^{15,19,27}$ Androsch et al. ${ }^{17-19}$ reported the direct generation of Form I' at large undercoolings in a butene-1/propylene copolymer with 11.4 mol\% comonomer. We note that a large amount of Form I was also obtained in this situation because of the simultaneous and fast Form II-Form I solid state transition in that condition. Therefore, it is plausible that the direct melt-crystallization of Form I' was induced by the earlier presence of Form I crystals by solid state transformation. Using a butene-1/ethylene copolymer, with high content of ethylene comonomer (9.8\%), Men et al. ${ }^{28,29,40}$ found that the Form I' crystals can be formed by melt memory effect which is independently from the original crystalline structure present before the melting stage. It was proposed that crystallizable and non-crystallizable chain sequences segregated during the crystallization process, constitute heterogeneities, with sizes that depend on melt temperature,, in which Form I' could nucleate. Although our results cannot exclude this mechanism, we note that the extent of segregation should be lower in our case, due to the lower content of ethylene comonomer (4.8\%). On the other hand, it clearly appears that Form I' can only be obtained in our system, when some Form I crystals (or latent nuclei) are still present in the melt. In conclusion, the mechanism of Form I' formation in the absence of selective nucleating substrates still needs investigation, and can depend on the comonomer content. 


\section{The competition between self-nucleation and cross-nucleation in PB1-ran-PE.}

Recently, it has been found that a given form of a polymer crystal can induce the nucleation of a different polymorph. This phenomenon is called cross-nucleation and has been reported in several semi-crystalline polymers, like polybutene-1, ${ }^{23,} 24$ polypropylene ${ }^{41}$ and polypivalolactone, ${ }^{42}$ amongst others.
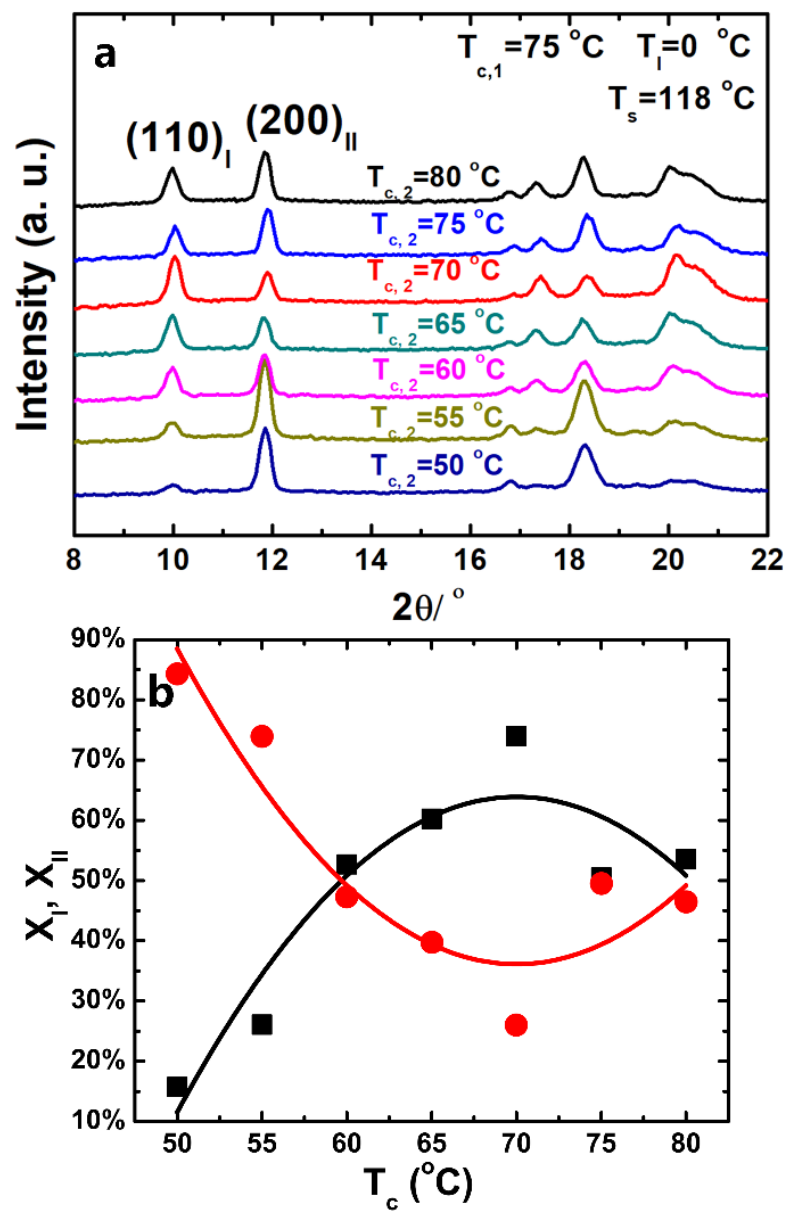

Figure 8. The competition between the cross-nucleation and self-nucleation behavior in PB1ran-PE. (a) The WAXD patterns of the self-nucleated sample crystallize at different temperatures. The $T_{c, 1}, T_{s}$ and $T_{l}$ values are 75,118 and $0{ }^{\circ} \mathrm{C}$, respectively (see the protocol in 
Figure 1d); (b) the fraction of Form I and Form II calculated by WAXD data.

Figure 8a shows WAXD data for the PB1-ran-PE sample after isothermal crystallization process at different temperatures (See experimental part, Figure 1d). All samples were first crystallized at $75{ }^{\circ} \mathrm{C}$ and then annealed at $0{ }^{\circ} \mathrm{C}$ and self-nucleated at $118{ }^{\circ} \mathrm{C}$ respectively. The contents of Form I' and Form II were estimated by the following equation ${ }^{43}$ :

$X_{I^{\prime}}=\frac{I(110)_{\mathrm{I}^{\prime}}}{I(110)_{\mathrm{I}^{\prime}}+R I(200)_{\mathrm{II}}}$

where $I(110)_{I^{\prime}}$ and $I(200)_{I I}$ are intensities of the (110) $)_{I^{\prime}}$ and (200) $)_{I I}$ reflections, respectively. The parameter $R$ in the equation is taken as $0.360 .67^{44,45}$, and it is used for correcting the different diffraction power of those crystallographic planes in trigonal and tetragonal crystal structures in Form I' and Form II. The trend of the polymorphic composition will likely depend on the competition between the nucleation and growth of each polymorph, which is strongly related to the crystallization temperature. In particular, the high growth rate of Form II at high temperatures and the low nucleation density of Form I' at low temperatures may determine the preference of the two crystal forms. In the intermediate crystallization temperature region (around $70{ }^{\circ} \mathrm{C}$ ), the maximum in Form I' content (and relative minimum of Form II) is possibly attributed to the enhanced Form I' formation by self-nucleation on Form I seeds. 


\section{Conclusions}

We have investigated the initial stage of the Form II to Form I transition of PB-1 and its PB1-ran-PE copolymer, using specially designed SN procedures. Different from other semi-crystalline polyolefins, the melt crystallized Form II can show an enhanced self-nucleation behavior when annealed at low temperatures $\left(T_{l}\right)$ for a short time, before partial melting.

For PB-1, the change in the re-crystallization behavior can be observed when the $T_{l}$ value is lower than $60{ }^{\circ} \mathrm{C}$, indicating the formation of "latent" Form I nuclei. If the $T_{l}$ temperature is lowered than $20^{\circ} \mathrm{C}$, a clear melting peak of Form I is observed, due to the growth of a small amount of Form I crystals during the subsequent heating process, as verified by temperature-resolved FTIR spectra.

In the copolymer case, Form II and Form I' can be nucleated due to the crossnucleation and self-nucleation phenomenon, respectively. The formation of Form I' can be enhanced when more Form I nuclei are present in the system, i.e., by annealing as decreasing $T_{l}$. Furthermore, the crystallization temperature is also found to play an important role in the competition of the formation of Form II and Form I', i. e., Form I' is favored by nucleation and growth at around $70{ }^{\circ} \mathrm{C}$.

\section{AUTHOR INFORMATION}

\section{Corresponding Authors}

*E-mail: alejandrojesus.muller@ehu.eus, djwang@iccas.ac.cn. 


\section{Notes}

The authors declare no competing financial interest.

\section{Acknowledgement}

Financial supports from the National Science Foundation of China (Grant No. U1510207) and the Key Program for Coal-based Science and Technology of Shanxi Province (MH-2014-08) are gratefully acknowledged. We would also like to thank the support of the Research and Innovation Staff Exchange (RISE) BIODEST H2020MSCA-RISE-2017-778092 project.

\section{Supporting Information}

Figure S1-S3: FT-IR and WAXD spectra of PB-1 and PB1-ran-PE samples during the cooling process, PLOM micrographs of PB-1 samples during the self-nucleating process, FT-IR spectra and the corresponding spectra of PB1-ran-PE during the heating process.

\section{References}

(1) Müller, A. J.; Michell, R. M.; Pérez, R. A.; Lorenzo, A. T. Successive Selfnucleation and Annealing (SSA): Correct design of thermal protocol and applications. European Polymer Journal 2015, 65, 132-154

(2) Michell, R. M.; Mugica, A.; Zubitur, M.; Müller, A. J., Self-Nucleation of Crystalline Phases Within Homopolymers, Polymer Blends, Copolymers, and 
Nanocomposites. In Polymer Crystallization I: From Chain Microstructure to Processing, Auriemma, F., Alfonso, G. C., DeRosa, C., Eds. Springer: Gewerbestrasse, 2017; Vol. 276, p 215.

(3) Maus, A.; Hempel, E.; Thurn-Albrecht, T.; Saalwachter, K. Memory effect in isothermal crystallization of syndiotactic polypropylene - role of melt structure and dynamics? The European physical journal. E, Soft matter 2007, 23 (1), 91-101

(4) Xu, J.; Ma, Y.; Hu, W.; Rehahn, M.; Reiter, G. Cloning Polymer Single Crystals through Self-seeding. Nat. Mater. 2009, 8 (4), 348-353

(5) Wang, B.; Tang, S.; Wang, Y.; Shen, C.; Reiter, R.; Reiter, G.; Chen, J.; Zhang, B. Systematic Control of Self-Seeding Crystallization Patterns of Poly(ethylene oxide) in Thin Films. Macromolecules 2018, 51 (5), 1626-1635

(6) Chen, X.; Wignall, G. D.; He, L.; Lopez-Barron, C.; Alamo, R. G. SANS Evidence of Liquid-Liquid Phase Separation Leading to Inversion of Crystallization Rate of Broadly Distributed Random Ethylene Copolymers. Macromolecules 2017, 50 (11), 4406-4414

(7) Reid, B. O.; Vadlamudi, M.; Mamun, A.; Janani, H.; Gao, H.; Hu, W.; Alamo, R. G. Strong Memory Effect of Crystallization above the Equilibrium Melting Point of Random Copolymers. Macromolecules 2013, 46 (16), 6485-6497

(8) Alfonso, G. C.; Scardigli, P. Melt Memory Effects in Polymer Crystallization. Macromol. Symp. 1997, 118, 323-328

(9) Alfonso, G. C.; Ziabicki, A. Memory Effects in Isothermal Crystallization II. Isotactic Polypropylene. Colloid and Polymer Science 1995, 273, 317-323 
(10)Lorenzo, A. T.; Arnal, M. L.; Sanchez, J. J.; Müller, A. J. Effect of annealing time on the self-nucleation behavior of semicrystalline polymers. Journal of Polymer Science Part B: Polymer Physics 2006, 44 (12), 1738-1750

(11) Sangroniz, L.; Barbieri, F.; Cavallo, D.; Santamaria, A.; Alamo, R. G.; Müller, A. J. Rheology of Self-nucleated Poly(e-caprolactone) Melts. European Polymer Journal 2018, 99, 495-503

(12)Luciani, L.; Seppala, J.; Lofgren, B. Polybutene-1: Its Preparation, Properties and Challenges. Prog. Polym. Sci 1988, 13, 37-62

(13) Natta, G.; Corradini, P.; Bassi, I. W. Crystal Structure of Isotactic Poly-alphaButene. Nuovo Cimento 1960, (1), 52-67

(14)Zhang, B.; Yang, D. C.; Yan, S. Direct Formation of Form I Poly(1-butene) Single Crystals from Melt Crystallization in Ultrathin Films. J. Polym. Sci, Part B: Polym. Phys. 2002, 40 (23), 2641-2645

(15) Wang, Z.; Dong, X.; Liu, G.; Xing, Q.; Cavallo, D.; Jiang, Q.; Müller, A. J.; Wang, D. Interfacial Nucleation in iPP/PB-1 Blends Promotes the Formation of Polybutene-1 Trigonal Crystals. Polymer 2018, 138, 396-406

(16) Woodward, A. E.; Morrow, D. R. Annealing of Polybutene-1 Single Crystals. Journal of Polymer Science Part a-2-Polymer Physics 1968, 6 (12PA), 1987-1997

(17) Stolte, I.; Fischer, M.; Roth, R.; Borreck, S.; Androsch, R. Morphology of Form I' Crystals of Polybutene-1 Formed on Melt-crystallization. Polymer 2015, 63, 30-33 (18) Stolte, I.; Cavallo, D.; Alfonso, G. C.; Portale, G.; Drongelen, M. v.; Androsch, R. Form I' crystal Formation in Random Butene-1/propylene Copolymers as Revealed 
by Real-time X-ray Scattering Using Synchrotron Radiation and Fast Scanning Chip Calorimetry. European Polymer Journal 2014, 60, 22-32

(19) Stolte, I.; Androsch, R. Comparative Study of the Kinetics of Non-isothermal Melt Solidification of Random Copolymers of Butene-1 with either Ethylene or Propylene. Colloid and Polymer Science 2014, 292 (7), 1639-1647

(20) Tashiro, K.; Hu, J.; Wang, H.; Hanesaka, M.; Saiani, A. Refinement of the Crystal Structures of Forms I and II of Isotactic Polybutene-1 and a Proposal of Phase Transition Mechanism between Them. Macromolecules 2016, 49 (4), 1392-1404 (21) Qiao, Y. N.; Wang, Q.; Men, Y. F. Kinetics of Nucleation and Growth of Form II to I Polymorphic Transition in Polybutene-1 as Revealed by Stepwise Annealing. Macromolecules 2016, 49 (14), 5126-5136

(22) Qiao, Y.; Men, Y. Intercrystalline Links Determined Kinetics of Form II to I Polymorphic Transition in Polybutene-1. Macromolecules 2017, 50 (14), 54905497

(23) Cavallo, D.; Gardella, L.; Portale, G.; Müller, A. J.; Alfonso, G. C. Kinetics of Cross-Nucleation in Isotactic Poly(1-butene). Macromolecules 2014, 47 (2), 870873

(24) Cavallo, D.; Gardella, L.; Portale, G.; Müller, A. J.; Alfonso, G. C. On Cross- and Self-nucleation in Seeded Crystallization of Isotactic Poly(1-butene). Polymer 2013, 54 (17), 4637-4644

(25) Cavallo, D.; Gardella, L.; Portale, G.; Müller, A. J.; Alfonso, G. C. Self-Nucleation of Isotactic Poly(1-butene) in the Trigonal Modification. Polymer 2014, 55 (1), 
(26) Cavallo, D.; Zhang, L.; Sics, I.; Alfonso, G. C.; Dumas, P.; Marco, C.; Ellis, G. The Morphology and Polymorphism of Self-nucleated Trigonal Isotactic Poly(1-butene) Studied by Synchrotron IR Microspectroscopy. Cryst. Eng. Comm 2016, 18 (5), 816-828

(27) Su, F. M.; Li, X. Y.; Zhou, W. M.; Zhu, S. S.; Ji, Y. X.; Wang, Z.; Qi, Z. M.; Li, L. B. Direct Formation of Isotactic Poly(1-butene) Form I Crystal from Memorized Ordered Melt. Macromolecules 2013, 46 (18), 7399-7405

(28) Wang, Y.; Lu, Y.; Zhao, J.; Jiang, Z.; Men, Y. Direct Formation of Different Crystalline Forms in Butene-1/Ethylene Copolymer via Manipulating Melt Temperature. Macromolecules 2014, 47 (24), 8653-8662

(29) Wang, Y.; Liu. Peiru; Lu, Y.; Men, Y. Mechanism of Polymorph Selection during Crystallization of Random Butene-1/Ethylene Copolymer. Chinese Journal of Polymer Science 2016, 34 (8), 1014-1020

(30)Xin, R.; Zhang, J.; Sun, X. L.; Li, H. H.; Ren, Z. J.; Yan, S. K. Polymorphic Behavior and Phase Transition of Poly(1-Butene) and Its Copolymers. Polymers 2018, 10 (5),

(31)Hsieh, E. T.; Randall, J. C. Ethylene-1-Butene Copolymer. 1. Comoner Sequence Distribution. Macromolecules 1982, 15, 353-360

(32) Fillon, B.; Wittmann, J. C.; Lotz, B.; Thierry, A. Self -Nucleation and Recrystallization of lsotactic Polypropylene ( $\alpha$ Phase) Investigated by Differential Scanning Calorimetry. Journal of polymer Science, Part B: Polymer 
Physics 1993, 31, 1383-1393

(33) Jiang, Q. H.; Zhao, Y.; Zhang, C. B.; Yang, J.; Xu, Y. Z.; Wang, D. J. In-situ Investigation on the Structural Evolution of Mesomorphic Isotactic Polypropylene in a Continuous Heating Process. Polymer 2016, 105 (22), 133-143

(34) M. Huang; X. Dong; D. Fu; W. Huang; L. Wu; Q. Huang; Wang, D. Effects of Ethylene Segments on the Crystallization and Tensile Properties of Biaxially Oriented Polypropylene. Acta Polymerica Sinica 2014, 4, 455-465

(35) Shi, J. Y.; Wu, P. Y.; Li, L.; Liu, T.; Zhao, L. Crystalline Transformation of Isotactic Polybutene-1 in Supercritical CO2 Studied by in-situ Fourier Transform Infrared Spectroscopy. Polymer 2009, 50 (23), 5598-5604

(36) Jiang, Q.; Zhao, Y.; Wang, D. Investigation on Microstructural Evolution of Mesomorphic Propylene/Ethylene Copolymer upon Thermal Treatment. Acta Polymerica Sinica 2017, 9, 1538-1548

(37) Fillon, B.; Thierry, A.; Wittmann, J. C.; Lotz, B. Self-Nucleation and Rescrystallization of Polymers. Isotactic Polypropylene, $\beta$ Phase: $\beta$ - $\alpha$ Convension and $\beta-\alpha$ Growth Transitions. J. Polym. Sci. Pt. B-Polym. Phys. 1993, 31 (10), 14071424

(38) Dai, P. S.; Cebe, P.; Capel, M. Thermal Analysis and X-ray Scattering Study of Metallocene Isotactic Polypropylene Prepared by Partial Melting. J. Polym. Sci. Pt. B-Polym. Phys. 2002, 40 (15), 1644-1660

(39) Varga, J.; Kargerkocsis, J. Direct Evidence of Row-nucleated Cylindritic Crystallization in Glass Fiber-reinforced Polypropylene Composites. Polymer 
Bulletin 1993, 30 (1), 105-110

(40)Lai, Y. Q.; Men, Y. F. Polymorph selection during crystallization of random copolymers. European Polymer Journal 2018, 101, 218-224

(41)Looijmans, S.; Menyhard, A.; Peters, G. W. M.; Alfonso, G. C.; Cavallo, D. Anomalous Temperature Dependence of Isotactic Polypropylene $\alpha$-on- $\beta$ CrossNucleation Kinetics. Crystal Growth \& Design 2017, 17 (9), 4936-4943

(42)Cavallo, D.; Galli, F.; Yu, L.; Alfonso, G. C. Cross-Nucleation between Concomitantly Crystallizing $\alpha$ - and $\gamma$-Phases in Polypivalolactone: Secondary Nucleation of One Polymorph on Another. Crystal Growth \& Design 2017, 17 (5), 2639-2645

(43)De Rosa, C.; Auriemma, F.; de Ballesteros, O. R.; Esposito, F.; Laguzza, D.; Di Girolamo, R.; Resconi, L. Crystallization Properties and Polymorphic Behavior of Isotactic Poly(1-Butene) from Metallocene Catalysts: The Crystallization of Form I from the Melt. Macromolecules 2009, 42 (21), 8286-8297

(44) Hu, J.; Tashiro, K. Relation between higher-order structure and crystalline phase transition of oriented isotactic polybutene-1 investigated by temperaturedependent time-resolved simultaneous WAXD/SAXS measurements. Polymer 2016, 90, 165-177

(45) Qiao, Y. N.; Wang, H.; Men, Y. F. Retardance of Form II to Form I Transition in Polybutene-1 at Late Stage: A Proposal of a New Mechanism. Macromolecules 2018, 51 (6), 2232-2239 
For Table of Contents use only

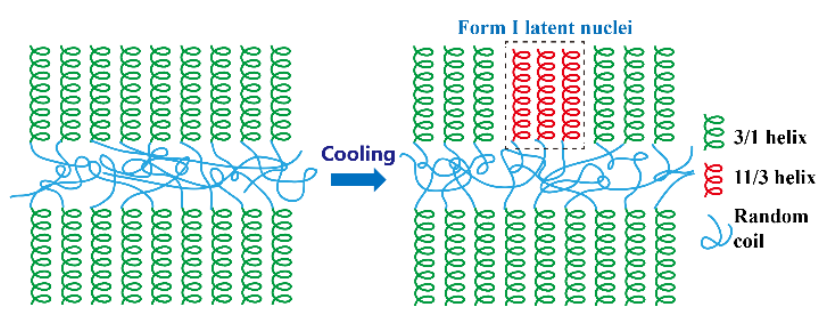

